\section{EXTREMELY SMALL AND LIGHTWEIGHT}

GC's NEW D-Light Duo is a cordless LED curing lamp which is extremely small and lightweight.

Its dual wavelength (400-480 nm) and reliable performance make it possible to cure all materials efficiently and in a very short time.

Manufactured in Switzerland from stainless steel with an autoclavable black light-guide, the other main features include: a 1200-1350 mW/ $\mathrm{cm}^{2}$ output; ability to deliver 97 ten second cycles with just 75 minutes recharging time; light weight (70 g) to reduce operator strain; slim and only $208 \mathrm{~mm}$ long to facilitate easy access and operator comfort.

D-Light Duo is supplied with a soft and translucent eye-protector shield, three years warranty on the device and one year warranty on the battery.

D-Light Duo is compatible with all the most popular light-cured products available in the UK including GC's comprehensive range of composites, glass ionomers and bonding materials.

For further information contact GC UK Ltd on 01908 218999, email info@gcukltd.co.uk or visit www. gceurope.com.

\title{
SURF THE CANAL WITH CONFIDENCE
}

WaveOne GOLD from DENTSPLY is the new generation of single-file reciprocating technology, specifically designed to increase the confidence of dental professionals in the safe use of NiTi instruments, whilst shortening the shaping time and covering a wider range of canal morphologies.

Keeping to the same philosophy of simplicity as the first generation WaveOne system, the same reciprocating motion, and one file suitable for $80 \%$ of cases, WaveOne GOLD takes efficiency to a new level, offering shorter shaping times and greater comfort. Constructed of nickel titanium, the file is repeatedly heat-treated and cooled after manufacturing, giving it a distinctive gold colour. This heat treatment significantly improves the file's strength and flexibility to negotiate a wider range of canal morphologies.
Wave0ne GOLD also boasts an extended range of file sizes. This allows for a greater range of canal morphologies to be covered. By introducing a medium 35 tip, four files, instead of the previous three, are now available; small \#20.07; primary \#25.07; medium \#35.06, and large \#45.05. The majority of cases (approx. 80\%) can be shaped with a single WaveOne GOLD Primary file, now 50\% more resistant to cyclic fatigue than its predecessor, which was more than twice as resistant as most standard rotary systems.

WaveOne GOLD is the perfect solution for those switching from hand files to a rotary system, those already using WaveOne, and those looking to simplify and save money over an existing rotary system.

For more information contact DENTSPLY on 08000723313 or visit dentsply.co.uk/gold.
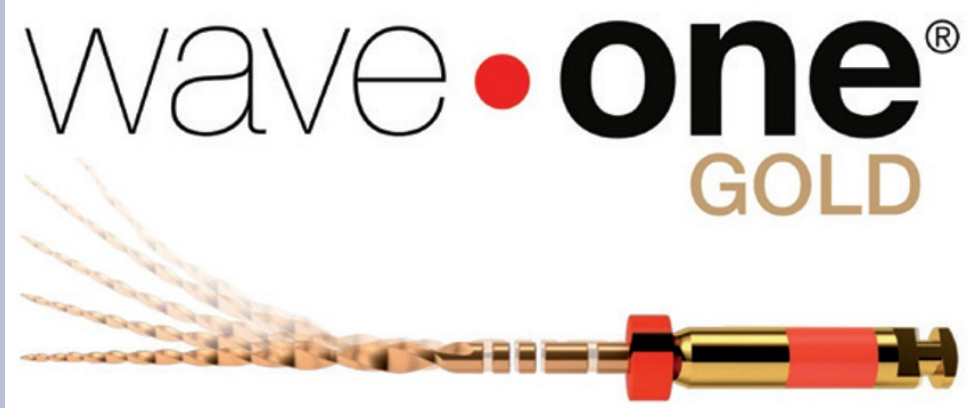

\section{HITTING MALOCCLUSION FOR SIX}

The nature of malocclusion, which requires first premolar tooth extraction, is an orthodontic problem affecting 20\% of patients in Europe. ${ }^{1}$ To help treat this section of the population Align Technology announced the release of Invisalign G6 a clinical innovation for first premolar extraction.

The announcement featured presentations on the biomechanical background to Invisalign G6 by John Morton, followed by Dr Francesco Garino who offered his initial experiences with first premolar extraction treatments and the possibilities offered by Invisalign G6 Innovations.

Invisalign G6 is engineered to improve clinical outcomes for the orthodontic treatment of severe crowding and bimaxillary protrusion which requires premolar extraction. Align's new advance provides vertical control and root parallelism using new SmartStage technology and SmartForce features which optimise the progression of tooth movements for first premolar extraction treatment planned for maximum anchorage.

Invisalign G6 for first premolar extraction is the culmination of perpetual clinical innovation at Align Technology. The new features comprise:

- New SmartStage programmed tooth movements that optimise the progression of tooth movements and provide aligner activation, which are engineered to eliminate unwanted tipping and unwanted anterior extrusion during retraction

- New SmartForce features which are designed to deliver the force systems necessary to achieve predictable tooth movements. These new features include Optimized Retraction Attachments, designed to work with SmartStage technology for effective bodily movement during canine retraction, with or without elastics, and new Optimized Anchorage Attachments, designed to work with SmartStage technology to maximise posterior anchorage.

Align Technology has also released ClinCheck Pro. The technology is designed to provide more precise control over final tooth position and includes interactive 3D controls which enable adjustments to be made to the position of individual teeth directly on the 3D model. Using this technology, Invisalign providers can visualise the effects on the whole dentition in real time.

For more information visit www.invisalign.co.uk.

1. Soh H, Sandham A, Chan Y H. Occlusal status in Asian male adults. Angle Orthod 2005; 75: 814-820. 